\title{
Resection of the vaginal vault for vaginal recurrence of cervical cancer after hysterectomy and brachytherapy
}

\author{
Akiko Abe*, Maki Matoda, Sanshiro Okamoto, Eiji Kondo, Kazuyoshi Kato, Kohei Omatsu, Kenji Umayahara, \\ Kuniko Utsugi and Nobuhiro Takeshima
}

\begin{abstract}
Background: We describe our experiences with vaginal vault resection for vaginal recurrence of cervical cancer after hysterectomy and radiotherapy. After operative treatment, the rate of vaginal vault recurrence of uterine cervical cancer is reported to be about 5\%. There is no consensus regarding the treatment for these cases.

Methods: Between 2004 and 2012, eight patients with vaginal vault recurrence underwent removal of the vaginal wall via laparotomy after hysterectomy and radiotherapy.

Results: The median patient age was 45 years (range 35 to 70 years). The median operation time was 244.5 min (range 172 to $590 \mathrm{~min}$ ), the median estimated blood loss was $362.5 \mathrm{~mL}$ (range 49 to 1,890 mL), and the median duration of hospitalization was 24.5 days (range 11 to 50 days). Two patients had intraoperative complications: a grade 1 bowel injury and a grade 1 bladder injury. The following postoperative complications were observed: one patient had vaginal vault bleeding, three patients developed vesicovaginal fistulae, and one patient had repeated ileus. Two patients needed clean intermittent catheterization. Local control was achieved in five of the eight cases.

Conclusions: Vaginal vault resection is an effective treatment for vaginal recurrence of cervical cancer after hysterectomy and radiotherapy. However, complications of this procedure can be expected to reduce quality of life. Therefore, this operation should be selected with great care.
\end{abstract}

Keywords: Cervical cancer, Vaginal recurrence, Radiotherapy, Vaginal vault resection, Postoperative complications

\section{Background}

Cervical cancer is the fourth most common cancer in women worldwide. In 2012, 528,000 cases of cervical cancer were diagnosed globally and 266,000 deaths were attributed to this disease [1]. In Japan, the incidence of early stage cervical cancer is increasing among younger women [2]. There are many long-term survivors of these cancers, and those who experience locoregional recurrence after initial surgery may require radiotherapy [3]. In most cases, radiotherapy can control vaginal vault recurrence $[4,5]$, but additional relapse is occasionally experienced. If it is feasible, surgical resection is generally considered for such cases. Vaginal vault resection is a technique that is applicable to rare clinical cases. Only a

\footnotetext{
* Correspondence: akiko06090111@yahoo.co.jp

Departments of Gynecology, Cancer Institute Hospital, 3-8-31 Ariake, Koto-ku, Tokyo 135-8550, Japan
}

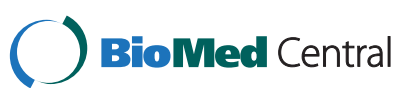

(c) 2015 Abe et al.; licensee BioMed Central. This is an Open Access article distributed under the terms of the Creative Commons Attribution License (http://creativecommons.org/licenses/by/4.0), which permits unrestricted use, distribution, and reproduction in any medium, provided the original work is properly credited. The Creative Commons Public Domain Dedication waiver (http://creativecommons.org/publicdomain/zero/1.0/) applies to the data made available in this article unless otherwise stated. few reports have described the efficacy of laparoscopic or vaginal vaginectomy [6,7]. To the best of our knowledge, this is the first study to report on vault resection performed via laparotomy. In the present study, we aimed to describe the efficacy and feasibility of vaginal vault resection by laparotomy for isolated small vaginal relapse of cervical cancer.

\section{Methods}

Eight patients were evaluated, each of whom experienced isolated vaginal relapse of uterine cervical cancer, and each of whom subsequently underwent vaginal vault resection by laparotomy at Cancer Institute Hospital (Tokyo, Japan) between 2004 and 2012. This research was performed with the approval of the Regional Ethics Committee for Clinical Study of the Cancer Institute Hospital, Tokyo, Japan, and in compliance with the Helsinki Declaration. The medical

ros 
records of these patients were reviewed retrospectively. Table 1 shows the patients' characteristics. Four patients were referred to our institute after prior hysterectomy, and four patients had undergone hysterectomy at our hospital. Hysterectomy was performed as simple extrafascial abdominal hysterectomy (four patients), vaginal hysterectomy (one patient), modified hysterectomy (two patients), or radical hysterectomy (one patient). In four patients, hysterectomy was combined with bilateral salpingooophorectomy, and in three patients, it was combined with pelvic lymphadenectomy.

Some patients had been treated with radiation using remote afterloading systems for positive margins of hysterectomy, and other patients had been treated for first vaginal vault recurrence after hysterectomy. After brachytherapy, some patients developed vaginal vault recurrence. Recurrences were treated with vaginal vault resection by laparotomy in eight cases. The intervals between brachytherapy and vaginal vault recurrences varied from 9 to 44 months. The patients were asked to indicate that they understood the nature of the surgical procedure, operative complications, and postoperative complications.

Surgical procedures were performed as follows (Figures 1 and 2). After bringing the patient into the Trendelenburg position, bilateral ureteral catheters and a Foley catheter were inserted under general anesthesia. Laparotomy was then performed, and exploration of the abdomen did not reveal other lesions in any of the cases. Adhesiolysis was performed between the rectum and the scar from the previous surgery. The pararectal and paravesical spaces were developed, and the surface of the levator ani muscle was exposed. Division between the posterior vaginal wall and the rectum was performed close to the muscular layer of the rectum, and this dissection plane was continued to the perineal body. The vaginal apex was identified using Hegar sounds and pulled via the apex. Ventral to the vagina, the plane of excision was placed in line with the limit of the muscular layer of the bladder. Subsequently, we resected the parametrium lateral to the vagina with the intention of

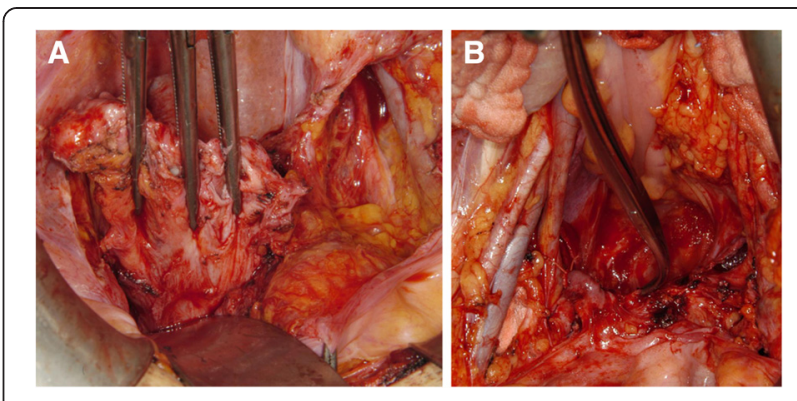

Figure 1 The vaginal apex identified using Hegar sounds and pulled via the apex (A) and pelvic appearance after vaginectomy (B).

preserving the nerve branches running from the inferior hypogastric plexus into the bladder. The vaginal wall was exfoliated via the transvaginal approach, and the vaginal vault was totally removed. Subsequently, the dissected end of the levator ani muscle was closed with sutures. We did not close the perineal opening, which was the dissecting line of the lower edge of the vagina. Finally, the bladder was filled with saline to check for leakage.

\section{Results}

Patient characteristics at the initial operation are summarized in Table 1 . The median patient age was 45 years (range 35 to 70 years), the median body mass index was 19.7 (range 16.8 to 24.5 ), and all patients met the criteria for American Society of Anesthesiologists physical status (PS) classification 1 to 2 . Three patients were diagnosed with carcinoma in situ: one patient was diagnosed with carcinoma in situ, one patient was diagnosed with adenosquamous cell carcinoma in situ, and one patient was diagnosed with adenocarcinoma in situ. Three patients had stage Ia disease: two had squamous cell carcinoma, and one had adenocarcinoma. Two patients had stage II disease: one had squamous cell carcinoma and one had adenosquamous cell carcinoma. Vaginal surgical margins

Table 1 Clinical features of the patients at the initial operation

\begin{tabular}{|c|c|c|c|c|c|c|c|c|}
\hline & Case 1 & Case 2 & Case 3 & Case 4 & Case 5 & Case 6 & Case 7 & Case 8 \\
\hline Age & 69 & 35 & 43 & 41 & 33 & 30 & 44 & 49 \\
\hline Initial stage & 0 & 0 & 0 & la & la & la & $\| a$ & $\| b$ \\
\hline Histology & AS in situ & $\mathrm{CIS}$ & AIS & SCC & $A C$ & SCC & SCC & AS \\
\hline Initial operation & $\mathrm{TAH}$ & $\mathrm{TAH}$ & $\mathrm{MRH}+\mathrm{BSO}+\mathrm{PLA}$ & $\mathrm{TAH}$ & $\mathrm{RH}+\mathrm{BSO}+\mathrm{PLA}$ & VTH & $\mathrm{MRH}+\mathrm{BSO}$ & $\mathrm{RH}+\mathrm{BSO}+\mathrm{PLA}$ \\
\hline Vaginal margin & Negative & Positive & Negative & NA & Negative & Positive & Positive & NA \\
\hline $\begin{array}{l}\text { Adaptation of radiation } \\
\text { treatment }\end{array}$ & Adjuvant & Recurrence & Recurrence & Adjuvant & Recurrence & Recurrence & Adjuvant & Recurrence \\
\hline $\begin{array}{l}\text { Interval between initial } \\
\text { operation and vaginectomy }\end{array}$ & $12 \mathrm{M}$ & $13 \mathrm{M}$ & $44 \mathrm{M}$ & $12 \mathrm{M}$ & $9 M$ & $38 M$ & $12 \mathrm{M}$ & $10 M$ \\
\hline
\end{tabular}

AS, adenosquamous cell carcinoma; CIS, carcinoma in situ; AIS, adenocarcinoma in situ; SCC, squamous cell carcinoma; AC, adenocarcinoma; TAH, total abdominal hysterectomy; $\mathrm{MRH}$, modified radical hysterectomy; $\mathrm{BSO}$, bilateral salpingo-oophorectomy; PLA, pelvic lymphadenectomy; $\mathrm{RH}$, radical hysterectomy; $\mathrm{VTH}$, vaginal total hysterectomy; NA, not available. 


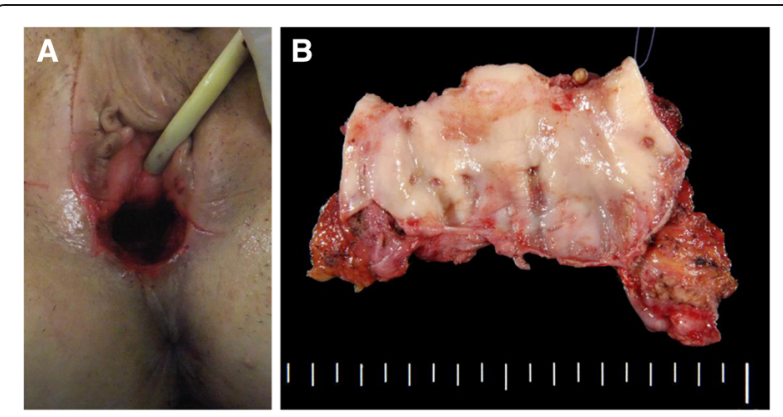

Figure 2 The vaginal appearance after the operation $(A)$ and the dissected vaginal specimen (B).

were positive in three cases, negative in three cases, and unavailable in two cases.

Table 2 presents operative data regarding resection of the vaginal vault and complications. The surgical margins were positive in four cases, negative in three cases, and unclear in one case, as caused by coagulation. The median operation time was $244.5 \mathrm{~min}$ (range 172 to $590 \mathrm{~min}$ ), and the median estimated blood loss was $362.5 \mathrm{~mL}$ (range 49 to $1,890 \mathrm{~mL}$ ). Bilateral ureteral catheters were inserted in seven patients. The Foley catheter was removed after a median of 7 days (range 3 to 20 days). Two patients needed clean intermittent catheterization after removal of the Foley catheter. The median hospital stay was 24.5 days (range 11 to 50 days).

With regard to postoperative complications, one patient experienced vaginal vault bleeding. Another patient experienced repeated ileus. Three patients developed vesicovaginal fistulae (Figure 3). For each of these patients, the Foley catheter was kept in place for 3 months, after which the fistula spontaneously closed. The median follow-up period after the resection of vault failure was 44.5 months (range 22 to 76 months). Regarding posttreatment surveillance, physical examinations were performed, with cytological investigation of the vaginal vault and transvaginal ultrasound (every 2 months for 2 years and every 4 months for another 3 years thereafter). After vaginal vault resection, three patients were disease-free and had good general condition. Five other patients developed recurrence: two developed distant metastasis, two developed locoregional failure, and one developed locoregional and distant metastasis. The median time to recurrence after vaginal resection was 10 months (range 2 to 22 months).

Two patients showed lymph node involvement after treatment for relapse. One of these patients had pelvic lymph node metastasis and was treated with radiotherapy. The other had para-aortic lymph node metastasis and was treated with chemotherapy. At the last follow-up, both patients were alive and well, with no evidence of disease. A patient with locoregional failure showed recurrence in the vagina, and pelvic exenteration and ureteroileostomy were performed. This patient died due to peritoneal dissemination 23 months after vaginal resection. A patient with locoregional and distant failure showed recurrence in the vulva after vaginal vault resection. The tumor was partially resected from the vulva. Furthermore, this patient developed multiple liver metastases 30 months later. With respect to postoperative complications, repeated ileus was managed with some difficulty. After a symptom was improved by fasting, this patient was treated with TS-1, resulting in the shrinkage of the recurrent tumors, and proton beam therapy was performed. Forty-five months after vaginal vault resection, the patient was alive with disease at the last follow-up.

\section{Discussion}

The rate of vaginal vault recurrence of stages I to II uterine cervical cancer after operative treatment has been reported to be $4.9 \%$ [8]. No consensus has been reached regarding the selection of multimodal treatment strategies. In general, radiotherapy is effective in terms of local control with vaginal vault recurrence. We treated first vaginal relapse with radiotherapy, and although 95\% patients were cured at our hospital, we sometimes observed second vaginal failures after providing radiotherapy for first vaginal relapse.

In a prior study, Berek et al. noted that intent-to-cure recurrent cervical carcinoma was the indication for pelvic exenteration after prior pelvic radiotherapy [9]. Although they found pelvic exenteration to be a feasible surgical procedure, it was associated with severe complications. Accordingly, Berek et al. described using pelvic exenteration as a last option for surgical therapy.

Panici et al. reported that the complications of pelvic exenteration include the development of ileus in $42 \%$ of patients (5/12), the requirement of re-laparotomy in $17 \%$ of patients (2/12), and the occurrence of leaks after intestinal anastomosis in $37.5 \%$ of patients (3/8) [7]. Panici et al.'s report further showed that the median operative time was $491.5 \mathrm{~min}$, the estimated blood loss was $2537.5 \mathrm{~mL}$, and the median hospital stay was 65.5 days. Another report has also shown similar results, including major and minor complication rates of $34 \%$ and $57 \%$, respectively [10]. Complete resection with exenteration is a significant prognostic factor for local control and survival, but it is also associated with high morbidity.

We have treated first vaginal relapse with radiotherapy and performed surgery for second vaginal failures. In consideration of morbidity, we have used surgery for resection of the vaginal vault, instead of using pelvic exenteration. The indication of resection for vault failure after hysterectomy and radiation included the following: 1) superficially carcinoma, 2) an interval of greater than 6 months between the initial operation and vault failure, 
Table 2 Clinical feature of the patients at vaginectomy

\begin{tabular}{|c|c|c|c|c|c|c|c|c|}
\hline & Case 1 & Case 2 & Case 3 & Case 4 & Case 5 & Case 6 & Case 7 & Case 8 \\
\hline Age & 70 & 36 & 48 & 44 & 35 & 36 & 46 & 54 \\
\hline Operative time (min), for vaginectomy & 180 & 590 & 219 & 172 & 270 & 316 & 191 & 442 \\
\hline Blood loss, mL & 250 & 1,890 & 49 & 150 & 365 & 490 & 360 & 760 \\
\hline Intraoperative complications & Rectal injury G1 & None & Bladder injury G1 & None & None & None & None & None \\
\hline Postoperative complications & Cystitis & $\begin{array}{l}\text { Vesicovaginal fistula } \\
\text { (POD33) }\end{array}$ & $\begin{array}{l}\text { Vesicovaginal fistula } \\
\text { (POD40), ileus, nephritis }\end{array}$ & None & None & Nephritis & $\begin{array}{l}\text { Vesicovaginal fistula } \\
\text { (POD19), nephritis }\end{array}$ & Stump bleeding \\
\hline Self-catheterization & No & No & No & No & + & No & No & + \\
\hline Margin of resection & Positive & Positive & Unclear & Positive & Negative & Positive & Negative & Negative \\
\hline Site of recurrence after colpectomy & PLN & Pelvic wall & Vulva & No & No & PAN & No & Vulva PLN \\
\hline Treatment for recurrence after colpectomy & Radiation & Exenteration & Vulvectomy & & & Chemotherapy & & $\begin{array}{l}\text { Vulvectomy + } \\
\text { lymphadenectomy }\end{array}$ \\
\hline Time to recurrence from colpectomy & $10 \mathrm{M}$ & $2 \mathrm{M}$ & $6 \mathrm{M}$ & & & $22 \mathrm{M}$ & & $19 M$ \\
\hline Final status & AWD & DOD & AWD & NED & NED & AWD & NED & AWD \\
\hline
\end{tabular}




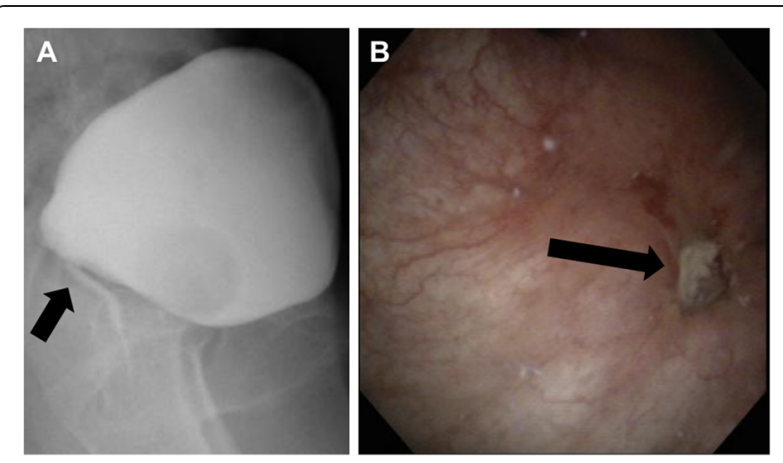

Figure 3 Urethrocystography showing a vesicovaginal fistula (arrow) (A, B).

and 3) consensus for the presence of both operative and postoperative complications. In this study, we have reported eight patients with vault failures who underwent vault resection.

Choi et al. reported four cases of laparoscopic upper vaginectomy for vaginal intraepithelial neoplasia and superficially invasive vaginal carcinoma after hysterectomy without radiotherapy [6]. Their operations were adapted for a recurrent lesion in the upper third of the vagina, and laparoscopic upper vaginectomy succeeded in controlling these cases without second vault failure.

Panici et al. reported cases in which vaginectomies were performed in patients who underwent radical hysterectomy without pelvic radiotherapy [7]. In this series, early postoperative complications were experienced by seven of the patients who had undergone vaginal vaginectomy: four patients developed vaginal scar abscess dehiscence, two experienced postoperative bleeding, and one developed a vesicovaginal fistula. Six subsequent complications were also observed: three patients developed vaginal stenosis, two patients developed urinary incontinence, and one patient developed ureteral stenosis. After vaginectomy, seven patients developed metastases, but no patient developed a second vaginal failure. Fiveyear overall and progression-free survival rates were $70.5 \%$ and $59.4 \%$, respectively. Panici et al. concluded that vaginal vaginectomy was feasible in selected patients. Their study's selection criteria were as follows: patients were included if they had vaginal lesions $<2 \mathrm{~cm}$ in size that were not juxtaposed to the rectum or bladder, and patients were excluded if they had prior radiation, prior nodal metastasis, palpable paravaginal invasion, or positive results in examinations for metastasis (including positron emission tomography (PET)). The criteria for performing a preoperative PET-computed tomography were the absence of both bulky tumor and distant disease.

In contrast to these previous reports, radiotherapy had been already performed in our study because of its good efficacy. At the second vault failure after radiotherapy, resection of the vault was performed via laparotomy. Total vaginectomy resulted in complications (grade 3 , $37.5 \% ; 3 / 8$ ) because of the adherence of the bladder and rectum after the first surgery and radiotherapy. Bladder filling was used to identify the altered anatomy after prior hysterectomy, as well as the induration of the vaginal vault leading to the bladder, vaginal apex, and border. Postoperative repeated cystitis and neurogenic bladder required longer hospitalization. Three patients developed vesicovaginal fistulae, which are associated with a high risk of severe complications. Vesicovaginal fistulae were managed conservatively, and fortunately, each of the patient's fistulae closed spontaneously. Technically feasible procedures could result in maintained bladder or bowel function.

\section{Conclusions}

Although this report is somewhat limited by the small number of patients, our results suggest that vaginectomy after radiotherapy is effective. In this series, local control was achieved in five cases. Since vaginal resection can be performed to achieve local control, this surgery is an option for vaginal vault recurrence, as is pelvic exenteration. However, an earlier study showed higher complication rates when this operation was performed after hysterectomy and brachytherapy. Because of the associated complications, vaginectomy should only be selected after carefully considering possible alternatives. For patients who have been selected carefully, vaginal resection is an acceptable treatment option.

Because of the rarity of the cases that we have considered, single-institution studies with large sample are likely to be infeasible. We suggest that data pooling is the most promising avenue for identifying optimal therapies and achieving other progress in this field. Such data pooling could come in the form of a review of all previously published case reports, case series, and small studies; the organization of prospective, multicenter case series; and/or the referral of all similar cases in Japan to a select group of medical institutes.

\section{Consent}

Written informed consent was obtained from the patient for the publication of this report and any accompanying images.

\section{Competing interests \\ The authors declare that they have no competing interests.}

\section{Authors' contributions}

TN planned the study. MM, OS, KE, KK, OK, and UK collected the cases. AA and TN wrote the draft. All authors read and approved the final manuscript.

\section{Authors' information}

Akiko Abe, Sanshiro Okamoto, Eiji Kondo, Kazuyoshi Kato, Kohei Omatsu, Kenji Umayahara, and Nobuhiro Takesima have MD and PhD degrees. Maki Matoda has an MD degree. 


\section{Acknowledgements}

We sincerely thank Dr. Takazawa Y (Cancer Institute Hospital).

Received: 17 September 2014 Accepted: 1 February 2015

Published online: 02 April 2015

\section{References}

1. International Agency for Research on Cancer. Cervical cancer estimated incidence, mortality and prevalence worldwide in 2012. World Health Organization; 2012. Available at: http://globocan.iarc.fr/Pages/ fact_sheets_cancer.aspx?cancer=cervix

2. Yamagami $\bar{W}$, Aoki $D$. Annual report of the committee on gynecologic oncology, the Japan Society of Obstetrics and Gynecology. J Obstet Gynaecol Res. 2014;40:338-48.

3. Thomas GM, Dembo AJ, Myhr T, Black B, Pringle JF, Rawlings G. Long-term results of concurrent radiation and chemotherapy for carcinoma of the cervix recurrent after surgery. Int J Gynecol Cancer. 1993;3:193-8.

4. Jobsen JJ, Leer JW, Cleton FJ, Hermans J. Treatment of locoregional recurrence of carcinoma of the cervix by radiotherapy after primary surgery. Gynecol Oncol. 1989;33:368-71.

5. Ito H, Shigematsu N, Kawada T, Kubo A, Isobe K, Hara R. Radiotherapy for centrally recurrent cervical cancer of the vaginal stump following hysterectomy. Gynecol Oncol. 1997;67:154-61.

6. Choi YJ, Hur SY, Park JS, Lee KH. Laparoscopic upper vaginectomy for post-hysterectomy high risk vaginal intraepithelial neoplasia and superficially invasive vaginal carcinoma. J Surg Oncol. 2013;11:126-30.

7. Panici PB, Manci N, Bellati F, Di Donato V, Marchetti C, De Falco C, et al. Vaginectomy: a minimally invasive treatment for cervical cancer vaginal recurrence. Int J Gynecol Cancer. 2009;19:1625-31.

8. Nakayama K, Teshima H, Hirai $Y$, Hasumi K, Masubuchi K. Stump recurrence after radical hysterectomy for patients with uterine cervical cancer (in Japanese). Nihon Sanka Fujinka Gakkai Zasshi. 1990;42:241-5.

9. Berek JS, Howe C, Lagasse LD, Hacker NF. Pelvic exenteration for recurrent gynecologic malignancy: survival and morbidity analysis of the 45-year experience at UCLA. Gynecol Oncol. 2005;99:153-9.

10. Ferenschild FTJ, Vermaas M, Vehoef C, Ansink AC, Kirkels WJ, Eggermont AMM, et al. Total pelvic exenteration for primary and recurrent malignancies. World J Surg. 2009;33:1502-8.

\section{Submit your next manuscript to BioMed Central and take full advantage of:}

- Convenient online submission

- Thorough peer review

- No space constraints or color figure charges

- Immediate publication on acceptance

- Inclusion in PubMed, CAS, Scopus and Google Scholar

- Research which is freely available for redistribution 